\title{
Effects of Flexible and Semirigid Lumbosacral Orthosis on Lower-Limb Joint Angles during Gait in Patients with Chronic Low Back Pain: A Cross-Sectional Study
}

\author{
Sang-Cheol Im, PT, PhD $\cdot$ Kyoung Kim, PT, $\mathrm{PhD}^{1 \dagger}$ \\ Department of Rehabilitation Sciences, Graduate School, Daegu University \\ ${ }^{1}$ Department of Physical Therapy, College of Rehabilitation Sciences, Daegu University
}

Received: August 10, 2021 / Revised: August 13, 2021 / Accepted: October 20, 2021

(C) 2021 J Korean Soc Phys Med

\section{| Abstract |}

PURPOSE: Lumbosacral orthosis (LSO) is often used to help manage low back pain because it is economical and effective. This study examined the effects of flexible and semirigid LSOs on the lower-limb joint angles in walking in patients with chronic low back pain.

METHODS: The effects of the lumbosacral orthosis during gait on the sagittal, frontal, horizontal planes and the change in lower limb angle were examined in fourteen chronic low back pain patients who walked without wearing a LSO, wearing a flexible LSO, and wearing a semirigid LSO in random order for three-dimensional motion analysis.

RESULTS: The flexion of the hip and knee joints decreased more significantly during walking with an LSO than without one. The genu valgum angles were reduced in the stance phase more during walking with an LSO than without one. The external rotation of the knee joints in the stance phase

$\dagger$ Corresponding Author : Kyoung Kim

kykim257@hanmail.net, https://orcid.org/0000-0003-4169-6852 This is an Open Access article distributed under the terms of the Creative Commons Attribution Non-Commercial License (http://creativecommons.org/licenses/by-nc/3.0) which permits unrestricted non-commercial use, distribution, and reproduction in any medium, provided the original work is properly cited. increased more during walking with an LSO than without one. CONCLUSION: The angles of the lower-limb joints of patients with chronic low back pain are affected by walking with an LSO, and the effects increased as the LSO stiffened.

Key Words: Gait, Kinematics, Low back pain, Lumbosacral orthosis, Motion analysis

\section{Introduction}

Low back pain is one of the most common musculoskeletal disorders in modern society [1]. Its chronic progression can induce a lower quality of life and increase medical costs, resulting in serious social and economic problems [2,3]. Continuous low back pain can hinder the movement of the pelvis, the spine, and the lower limbs during walking [4], and patients with chronic low back pain exhibit asymmetric gait patterns because of pain and hypoesthesia in the lower-back area [5]. The asymmetric gait of patients with chronic low back pain is reported to occur as they perceive inadequate temporal and spatial information because of a decreased peripheral feedback mechanism caused by pain [6]. Patients with low back pain tend to walk rigidly, with their bodies fixed and pelvic movements either increased or decreased to avoid pain 
$[7,8]$. Thus, prior research has highlighted the need for further studies to develop methods of functional training and gait rehabilitation to improve the abnormal gait patterns of these patients $[9,10]$.

Spinal orthosis is generally designed to evoke motor sensations and provide complete contact, three-point pressure, endpoint control, and high pressure [11]. Wearing a spinal orthosis can provide mechanical support and psychological stability to patients with low back pain, thereby reducing the discomfort that they experience [12]. In particular, a lumbosacral orthosis (LSO) can limit the range of motion of the hip bones and help maintain the stability of the musculoskeletal system while also enhancing the stability of the spine in particular [13]. According to several studies, patients with chronic low back pain have impaired proprioceptive sense of the lumbar region [14-16]. This impaired perception can cause low back pain that may result in or sustain joint instability [17]. Hence, wearing an LSO may be beneficial in such patients [18]. Wearing an LSO increases the pressure exerted on the skin, which provides additional afferent sensory information to the central nervous system via mechanical receptors [19], which may ultimately improve the proprioceptive sense of the lumbar region [20]. Wearing an LSO increases the mechanical rigidity, which may decrease the structural load of the spine by limiting the lumbar movement [21]. To treat low back pain, patients are often advised to wear an appropriate orthosis and utilize a tailor-made exercise therapy program to maintain their bodies' flexibility and muscular strength [22].

Previous studies on spinal orthosis have typically investigated the movements of the spine and trunk [23], their effects on the total movement of the spine [24], and the amount of spinal limitations provided in the maximum flexion/extension/rotation postures of the torso [23-27]. For example, Buchalter et al. [25] reported that wearing thoracolumbosacral orthosis limited $69 \%$ of the flexion/extension, $94 \%$ of the lateral flexion of the hip bones, $49 \%$ of the flexion/extension, $38 \%$ of the lateral flexion of the spine, and $60 \%$ of the total trunk rotation. Fiddler and Plasmans [23] reported that wearing LSOs limited movements between the 4th and 5th lumbar vertebrae by an average of $32 \%$ and between the 5th lumbar vertebra and the 1st sacral vertebra by an average of $70 \%$. In several recent studies, however, it has been suggested that the limited angle of lumbar spine rotation from wearing spinal orthosis was not significant because the rotation was also limited under conditions of not wearing spinal orthosis [11].

In any case, this class of device, including LSOs, generally aims to support both the spine and pelvis, directly affecting the spinal movement and lower-limb kinematics. According to Kramers-de Quervain et al. [28], female patients with adolescent scoliosis showed decreased pelvic angles on the frontal plane and trunk rotation on the horizontal plane when they wore spinal orthosis. Recent studies examined the effect of wearing LSOs on gait, including one utilizing 21 patients with adolescent idiopathic scoliosis who wore flexible or rigid LSOs during flat-ground walking. Both groups (using either flexible or rigid LSOs) showed significant decreases in pelvic ascending/descending obliquity and abduction/adduction of the hip joints but no changes in the knee or ankle joint angles [29].

Konz et al. [30] examined 10 healthy subjects with and without fiberglass body jackets, similar to thoracolumbosacral orthosis, walking at five speeds and reported a significant decrease in the peak and trough of pelvic obliquity and rotation by spinal limitation only at the highest speed. The peak and trough of the hip joint abduction/adduction decreased significantly at all speeds with spinal limitation, but that of the hip joint flexion/extension of the joints increased significantly only at slow and very slow speeds [30]. Song et al. [31] compared the effects of restricting trunk movement on the gait patterns when healthy subjects wore rigid thoracolumbosacral orthosis. The results showed that the step width of the group wearing orthosis decreased 
significantly during flat-ground walking and that the flexion of the ankles on the sagittal plane increased during stair climbing.

Spinal orthosis is commonly used to treat various conditions affecting the spine. On the other hand, orthosis affects the spinal movement movements of the pelvis and lower limbs during walking because both the spine and the pelvis are involved. Most previous studies on the effects of spinal orthosis on gait have dealt with healthy people or patients with scoliosis; little research has been conducted on its effects regarding the gait of patients with chronic low back pain. In particular, there is a lack of research addressing the walking patterns when wearing LSOs with different degrees of stiffness. In addition, although there are studies reporting that semirigid LSO increases the lumbar stiffness more than flexible LSO [32], some studies reported no difference between flexible LSO and semirigid LSO [33]. As conflicting research results have been reported on the difference in LSO stiffness, additional research on the difference in LSO stiffness is needed.

This study examined this specific issue: the effects of wearing LSOs providing varying levels of support for the lower-limb kinematics of patients with chronic low back pain in gait, providing basic data for clinical prescriptions and adaptation training for orthosis. It was hypothesized that the differences in LSO stiffness would affect the lower extremity kinematics during walking in chronic low back pain patients.

\section{Methods}

\section{Participants}

The subjects were initially 14 patients with chronic low back pain who visited a spine hospital in Daegu, Republic of Korea, and met the criteria described below. G-power 3.1.9.4 was used to determine the sample size using a previous study with the same design as a reference [30]. Accordingly, the sample size of 14 patients was necessary to achieve an $80 \%$ probability (effect size $=.36, \alpha=.05$, power $=.80$ ). All subjects received a sufficient explanation of the purpose and methods of the study before voluntarily agreeing to participate in this experiment. The inclusion criteria were patients with low back pain for at least three months, could walk independently without assisting devices, and showed stable medical conditions. Those with neurological symptoms that may disrupt walking or operation history in the lumbosacral site were excluded. This study was approved by the institutional review board of Daegu University (approval no. 1040621-201702-HR005-02) and was conducted in accordance with the Declaration of Helsinki.

\section{Experimental Procedure}

The researchers produced a comfortable environment so that the subjects did not feel unease in indoor temperature and surrounding circumstances. The subjects wore sleeveless T-shirts and short pants made from a lightweight material that clung to their bodies so it would not disturb the measurements. Before measuring the gait, the same examiners measured the anthropometric data of the subjects to identify their general characteristics (age, height, weight, leg length, and foot length). All subjects underwent the experiment without wearing shoes, and they walked comfortably and naturally on a straight $10 \mathrm{~m}$ path painted on a flat, solid floor at their usual speed. To measure their natural gait, they were asked to walk a path five times as a warm-up procedure. The researchers asked them to focus continually on a dot painted on the wall ahead while walking.

To determine the changes based on the stiffness of the orthosis, the subjects conducted three types of walking: walking without orthosis (Condition 1), walking with flexible LSOs (Condition 2), and walking with semirigid LSOs (Condition 3). The researchers performed motion analyses for all three conditions for each subject. To exclude the learning effects, these three events were undertaken 


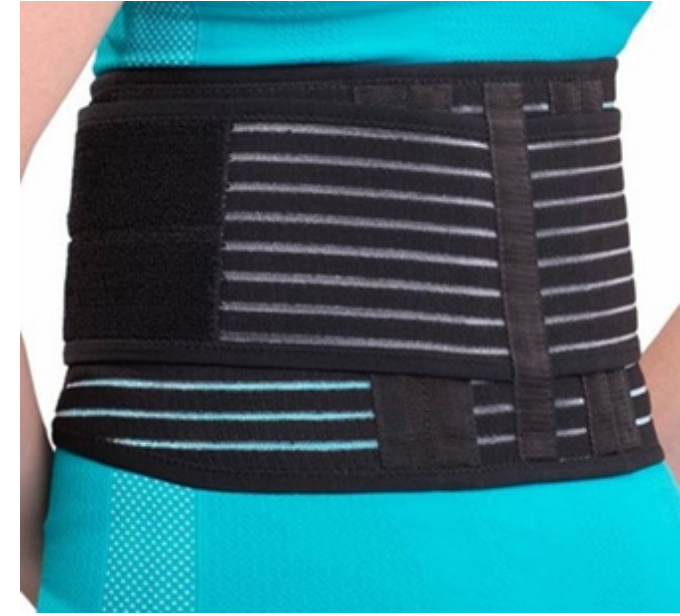

Fig. 1. Flexible LSO (REDIX-K210).

in random order. The researchers did not inform the subjects of the measurement period so that they would not perceive either the moment at which they were first being measured or when the monitoring ended. The researchers only started measuring the gait after the first five steps. A $30 \mathrm{~s}$ break between each walk and a 5 min rest between gait conditions were provided to reduce measurement errors. The researchers measured each walk under the three conditions five separate times and analyzed the most natural walk during which the force-measuring board was accurately touched.

The anterior part of the orthosis should be located from below the xiphoid process to just above the symphysis pubis, and the top of the rear section should be located just below the scapular inferior angle. The devices used in this study were LSOs (Acetech, Korea); these offthe-shelf products came in three sizes (large, medium, and small) and were used according to the body types of the subjects. The orthosis was applied consistently by the researchers and was tightened as far as the subject could endure.

1) Flexible LSOs (REDIX-K210, Acetech, Korea). This is a modified version of flexible orthosis into which rear support is inserted. It is made of functional

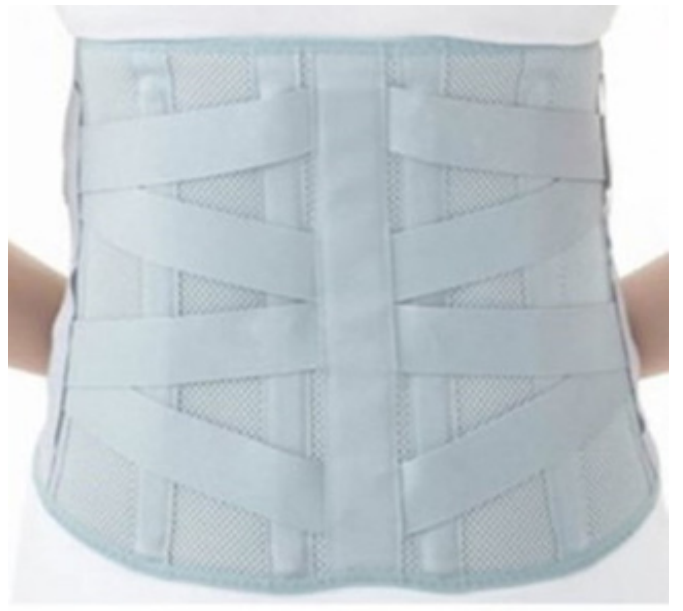

Fig. 2. Semirigid LSO (REDIX-L350).

material with advanced ventilation and Velcro fasteners that make it conveniently adjustable to a wide variety of body types (Fig. 1).

2) Semirigid LSOs (REDIX-L350, Acetech, Korea). This type of system has a rear plate for stable support and uses reinforced frames inserted front and back to limit flexion and extension, providing firm support with a front-opening structure and auxiliary belt connections (Fig. 2).

\section{Measurement Methods}

The measurements were taken in the motion analysis laboratory of a spine hospital located in Daegu. Three-dimensional walking data were recorded as subjects walked a $10 \mathrm{~m}$ path at the center of the lab. The researchers used the Orthotrak 6.5.1 program (Orthotrak, Motion Analysis, USA), computers with EvaRT 5.0.3 (EvaRT, Motion Analysis, USA), and six Eagle system infrared cameras (Eagle system, Motion Analysis, USA) that were connected to the computers and fixed on walls. The data were processed using EvaRT 5.0.3 and Orthotrak 6.5.1. Before the experiment, calibrations were performed to correct the errors that might occur in the infrared cameras, and a static check was then carried out in which barefoot 


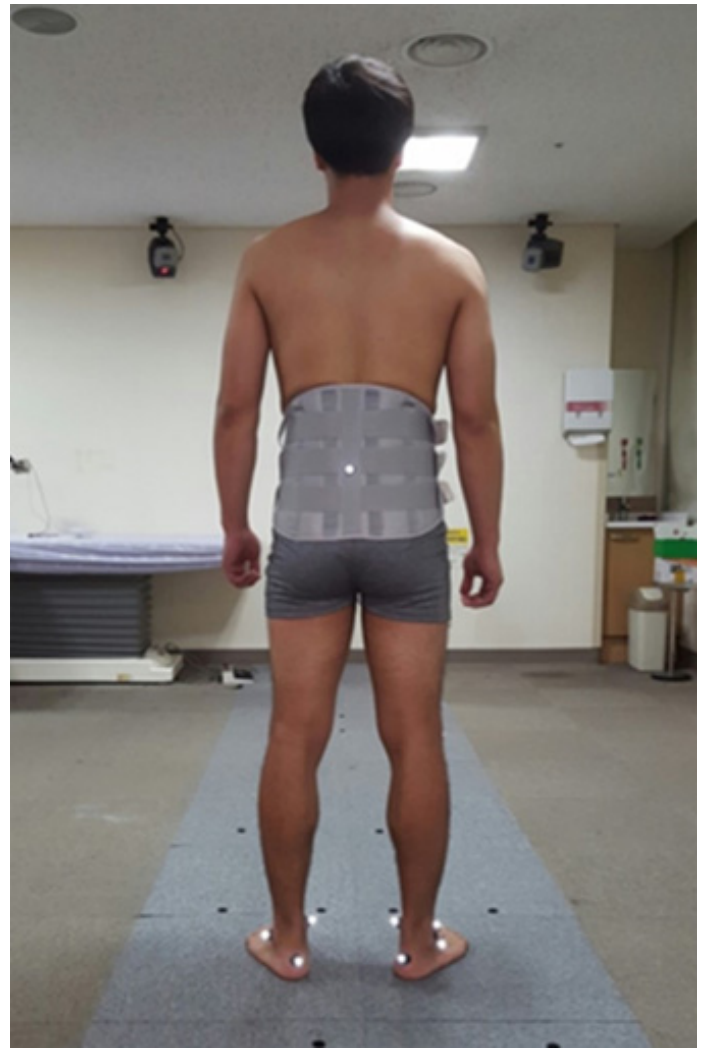

Fig. 3. Posterior view of a subject wearing an LSO and reflective markers.

subjects stood straight, and the location of each joint was confirmed on the computer's screen. During this check, the subjects stood on a force plate for $2 \mathrm{~s}$ with reflective markers (25 $\mathrm{mm}$ in diameter) attached to the pelvis and lower limbs. One examiner attached the markers to segments of the lower limbs and pelvis according to the
Helen Hayes marker set, a plug-in gait marking system.

Nineteen markers were attached to the sacrum, the left and right anterior superior iliac spines, the left and right thighs' center, the lateral epicondyles of the left and right knee joints, the left and right tibias' center, the lateral malleolus of the left and right ankle joints, the head of the left and right second metatarsal bone, and the back of the left and right heel bones. For areas where markers were prone to fall, they were fixed with Kinesio tape that does not reflect light (Fig. 3). After the static test, the length from the lateral malleolus of each ankle to the markers [left and right anterior superior iliac spine (ASIS), sacrum] covered by the lumbosacral orthosis was measured in the upright standing position, and the distance between each marker was measured using a flexible ruler. Using this measured distance/length, the markers were reattached to the same location in other lumbosacral orthosis conditions. For experimental consistency, the same inspectors with 10 or more years of motion analysis experience measured the subjects' bodies, attached the markers, and conducted other measurement procedures for the entire experiment. Table 1 lists the kinematic data of each joint that was classified according to the positive and negative values.

\section{Statistical Analysis}

The general characteristics of the subjects were analyzed using descriptive statistics. The collected data were processed statistically using SPSS 18.0 (IBM Corp.,

Table 1. Kinematic Variables Under Evaluation

\begin{tabular}{ccccc}
\hline Joint & Angle & Sagittal plane & Frontal plane & Horizontal plane \\
\hline \multirow{2}{*}{ Hip } & Positive & Flexion & Adduction & Internal rotation \\
& Negative & Extension & Abduction & External rotation \\
\hline \multirow{2}{*}{ Knee } & Positive & Flexion & Varus & Internal rotation \\
& Negative & Extension & Valgus & External rotation \\
\hline \multirow{2}{*}{ Ankle } & Positive & Dorsiflexion & Inversion & Internal rotation \\
& Negative & Plantarflexion & Eversion & External rotation \\
\hline
\end{tabular}


Table 2. General and Physical Characteristics of the Subjects $(n=14)$

\begin{tabular}{ccc}
\hline Variable & Mean $\pm \mathrm{SD}$ & Range \\
\hline Gender (male/female) & $6 / 8$ & \\
Age (years) & $39.93 \pm 11.76$ & $26-59$ \\
Height $(\mathrm{cm})$ & $168.36 \pm 7.19$ & $158-180$ \\
Weight $(\mathrm{kg})$ & $67.29 \pm 15.87$ & $52-108$ \\
Leg length $(\mathrm{cm})$ & $88.32 \pm 4.56$ & $79-96$ \\
Foot length $(\mathrm{cm})$ & $24.43 \pm 1.36$ & $23-27$ \\
Foot width $(\mathrm{cm})$ & $9.15 \pm .96$ & $8.2-11.2$ \\
Knee width $(\mathrm{cm})$ & $11.32 \pm 1.03$ & $9.8-13.5$ \\
Ankle width $(\mathrm{cm})$ & $6.88 \pm .71$ & $6-8.5$ \\
Onset (months) & $31.00 \pm 31.29$ & $4-120$ \\
ODI $(\%)$ & $18.43 \pm 4.16$ & $12-26$ \\
\hline
\end{tabular}

Armonk, USA) for Windows. A normality test was conducted using the KolmogorovSmirnov test. Repeated measures analysis of variance was used to determine the differences across the three gait conditions. A least-squares difference was used as a posttest to describe the differences between the gait conditions. All statistical significance levels were set to $\mathrm{p}=.05$.

\section{Results}

\section{General Characteristics of the Subjects}

Fourteen subjects participated in this study, whose mean age was 39.93 years. The mean onset duration was 31 months; the mean Visual Analog Scale (VAS) score was 3.07, and the Oswestry Disability Index (ODI) was $18.43 \%$. Table 2 lists the age, height, weight, leg length, foot length, foot diameter, knee diameter, and ankle diameter of the subjects.

\section{Analysis of the Kinematic Data}

Table 3 lists the angle values (mean \pm standard deviation [SD]) of the hip, knee, and ankle joints at the initial contact phase, midstance phase, preswing phase, and midswing phase during gait on the sagittal, frontal and horizontal planes based on gait conditions. There was a significant difference in the hip joint angle at the initial contact, midstance phase, toe-off, and midswing phase of the sagittal plane $(\mathrm{p}<.05)$. Post hoc analysis revealed a significant difference between Condition 1 and Conditions 2 and 3 in the initial contact, midstance phase, and toe-off. There was a significant difference between all walking conditions in the midswing phase $(\mathrm{p}<.05)$.

A significant difference in knee joint angle was observed at the midstance phase and midswing phase of the sagittal plane $(\mathrm{p}<.05)$. Post-hoc analysis revealed a significant difference between Condition 1 and Conditions 2 and 3 in the midstance phase and a significant difference only between Conditions 1 and 3 in the midswing phase (p $<.05)$. A significant difference was observed in the initial contact, midstance phase, and toe-off in the frontal plane $(\mathrm{p}<.05)$. Post-hoc analysis, there was a significant difference only between Conditions 1 and 3 in the initial contact. There was a significant difference between Condition 1 and Conditions 2 and 3 in the midstance phase $(\mathrm{p}<.05)$. A significant difference was noted between all walking conditions in toe-off $(p<.05)$. There was a significant difference in the midstance phase and toe-off of the horizontal plane $(\mathrm{p}<.05)$. A significant difference 
Table 3. Comparison of Joint Angles Across Gait Conditions

\begin{tabular}{|c|c|c|c|c|c|}
\hline Variable & Condition 1 (without LSO) & Condition 2 (flexible LSO) & Condition 3 (semirigid LSO) & $\mathrm{p}$ & Post-hoc \\
\hline H1 & $30.13 \pm 4.79$ & $26.02 \pm 5.72$ & $24.58 \pm 7.12$ & $.006^{*}$ & $\mathrm{C} 1>\mathrm{C} 2, \mathrm{C} 1>\mathrm{C} 3$ \\
\hline $\mathrm{H} 2$ & $8.04 \pm 5.64$ & $3.47 \pm 7.51$ & $2.91 \pm 8.15$ & $.005^{*}$ & $\mathrm{C} 1>\mathrm{C} 2, \mathrm{C} 1>\mathrm{C} 3$ \\
\hline $\mathrm{H} 3$ & $.08 \pm 7.25$ & $-3.12 \pm 8.35$ & $-3.56 \pm 8.14$ & $.011^{*}$ & $\mathrm{C} 1>\mathrm{C} 2, \mathrm{C} 1>\mathrm{C} 3$ \\
\hline $\mathrm{H} 4$ & $28.61 \pm 5.20$ & $26.05 \pm 5.64$ & $24.05 \pm 5.80$ & \multicolumn{2}{|c|}{$.009^{*} \mathrm{C} 1>\mathrm{C} 2, \mathrm{C} 2>\mathrm{C} 3, \mathrm{C} 1>\mathrm{C} 3$} \\
\hline $\mathrm{H} 5$ & $-.38 \pm 4.65$ & $-.82 \pm 4.64$ & $-1.36 \pm 4.97$ & \multicolumn{2}{|l|}{.643} \\
\hline H6 & $3.22 \pm 3.86$ & $1.72 \pm 4.23$ & $2.13 \pm 2.89$ & \multicolumn{2}{|l|}{.310} \\
\hline $\mathrm{H} 7$ & $-4.61 \pm 3.60$ & $-5.47 \pm 4.02$ & $-5.15 \pm 3.26$ & \multicolumn{2}{|l|}{.532} \\
\hline $\mathrm{H} 8$ & $-2.60 \pm 4.27$ & $-3.24 \pm 3.69$ & $-3.60 \pm 4.02$ & \multicolumn{2}{|l|}{.617} \\
\hline H9 & $6.28 \pm 6.08$ & $6.30 \pm 6.22$ & $5.33 \pm 8.83$ & \multicolumn{2}{|l|}{.521} \\
\hline $\mathrm{H} 10$ & $2.86 \pm 5.32$ & $2.61 \pm 5.72$ & $2.78 \pm 6.94$ & \multicolumn{2}{|l|}{.967} \\
\hline H11 & $-4.57 \pm 5.27$ & $-3.95 \pm 5.40$ & $-3.78 \pm 6.30$ & \multicolumn{2}{|l|}{.770} \\
\hline H12 & $1.40 \pm 4.91$ & $1.40 \pm 4.91$ & $.79 \pm 4.60$ & \multicolumn{2}{|l|}{.502} \\
\hline K1 & $14.19 \pm 4.36$ & $11.78 \pm 4.40$ & $11.13 \pm 5.59$ & \multicolumn{2}{|l|}{.098} \\
\hline $\mathrm{K} 2$ & $16.44 \pm 4.87$ & $12.67 \pm 5.48$ & $12.59 \pm 5.95$ & \multicolumn{2}{|r|}{$\mathrm{C} 1>\mathrm{C} 2, \mathrm{C} 1>\mathrm{C} 3$} \\
\hline K3 & $43.93 \pm 4.07$ & $42.87 \pm 5.20$ & $41.93 \pm 4.88$ & \multicolumn{2}{|l|}{.222} \\
\hline K4 & $56.95 \pm 5.34$ & $55.52 \pm 5.10$ & $53.42 \pm 6.21$ & \multicolumn{2}{|c|}{$.021^{*} \quad \mathrm{C} 1>\mathrm{C} 3$} \\
\hline $\mathrm{K} 5$ & $.74 \pm 2.83$ & $1.54 \pm 3.91$ & $1.83 \pm 4.25$ & \multicolumn{2}{|c|}{$.047^{*} \quad \mathrm{C} 1<\mathrm{C} 3$} \\
\hline K6 & $.31 \pm 3.56$ & $1.51 \pm 4.28$ & $1.62 \pm 4.84$ & \multicolumn{2}{|c|}{$.019^{*} \quad \mathrm{C} 1<\mathrm{C} 2, \mathrm{C} 1<\mathrm{C} 3$} \\
\hline K7 & $-1.73 \pm 4.11$ & $-.76 \pm 3.84$ & $-.20 \pm 4.06$ & \multicolumn{2}{|c|}{$.010^{*} \mathrm{C} 1<\mathrm{C} 2, \mathrm{C} 2<\mathrm{C} 3, \mathrm{C} 1<\mathrm{C} 3$} \\
\hline K8 & $1.79 \pm 4.14$ & $1.64 \pm 4.53$ & $2.07 \pm 3.70$ & \multicolumn{2}{|l|}{.622} \\
\hline K9 & $-17.35 \pm 9.84$ & $-17.57 \pm 9.54$ & $-18.41 \pm 9.20$ & \multicolumn{2}{|l|}{.319} \\
\hline K10 & $-10.96 \pm 11.42$ & $-12.04 \pm 11.53$ & $-12.77 \pm 11.39$ & \multicolumn{2}{|c|}{$.041^{*} \quad \mathrm{C} 1>\mathrm{C} 3$} \\
\hline K11 & $-15.44 \pm 5.15$ & $-17.10 \pm 5.54$ & $-17.26 \pm 6.01$ & \multicolumn{2}{|c|}{$.004^{*} \mathrm{C} 1>\mathrm{C} 2, \mathrm{C} 2>\mathrm{C} 3, \mathrm{C} 1>\mathrm{C} 3$} \\
\hline K12 & $-19.25 \pm 9.63$ & $-19.34 \pm 10.36$ & $-19.10 \pm 10.46$ & \multicolumn{2}{|l|}{.939} \\
\hline A1 & $4.11 \pm 2.25$ & $3.96 \pm 2.69$ & $4.03 \pm 1.68$ & \multicolumn{2}{|l|}{.961} \\
\hline $\mathrm{A} 2$ & $13.81 \pm 3.23$ & $13.32 \pm 2.99$ & $13.70 \pm 3.30$ & \multicolumn{2}{|l|}{.400} \\
\hline $\mathrm{A} 3$ & $-4.19 \pm 6.24$ & $-5.16 \pm 5.64$ & $-4.51 \pm 6.20$ & \multicolumn{2}{|l|}{.719} \\
\hline A4 & $5.31 \pm 3.62$ & $5.22 \pm 2.89$ & $5.31 \pm 3.96$ & \multicolumn{2}{|l|}{.993} \\
\hline A5 & $9.49 \pm 7.67$ & $10.14 \pm 7.76$ & $10.30 \pm 7.18$ & \multicolumn{2}{|l|}{.494} \\
\hline A6 & $6.16 \pm 7.03$ & $7.22 \pm 7.58$ & $6.88 \pm 7.46$ & \multicolumn{2}{|l|}{.507} \\
\hline A7 & $14.29 \pm 8.00$ & $14.70 \pm 8.46$ & $14.83 \pm 8.26$ & \multicolumn{2}{|l|}{.837} \\
\hline A8 & $9.72 \pm 5.92$ & $10.26 \pm 6.06$ & $10.54 \pm 6.10$ & .443 & \\
\hline A9 & $1.31 \pm 8.69$ & $2.26 \pm 7.47$ & $2.82 \pm 6.59$ & .156 & \\
\hline $\mathrm{A} 10$ & $1.39 \pm 6.55$ & $2.05 \pm 6.60$ & $2.25 \pm 6.01$ & .351 & \\
\hline A11 & $12.63 \pm 7.90$ & $13.17 \pm 7.36$ & $13.04 \pm 7.76$ & .873 & \\
\hline $\mathrm{A} 12$ & $3.70 \pm 6.40$ & $4.79 \pm 6.00$ & $4.34 \pm 6.95$ & .385 & \\
\hline
\end{tabular}

${ }^{*} \mathrm{p}<.05$

H1: hip flexion/extension at initial contact; H2: hip flexion/extension at midstance phase; H3: hip flexion/extension at toe-off; H4: hip flexion/extension at midswing phase; H5: hip adduction/abduction at heel strike; H6: hip adduction/abduction at midstance phase; H7: hip adduction/abduction at toe-off; H8: hip adduction/abduction at midswing phase; H9: hip internal/external rotation at heel strike; H10: hip internal/external rotation at midstance phase; H11: hip internal/external rotation at toe-off; H12: hip internal/external rotation at midswing phase; K1: knee flexion/extension at heel strike; K2: knee flexion/extension at midstance phase; K3: knee flexion/extension at toe-off; K4: knee flexion/extension at midswing phase; K5: knee varus/valgus at heel strike; K6: knee varus/valgus at midstance phase; K7: knee varus/valgus at toe-off; K8: knee varus/valgus at midswing phase; K9: knee internal/external rotation at heel strike; K10: knee internal/external rotation at midstance phase; K11: knee internal/external rotation at toe-off; K12: knee internal/external rotation at midswing phase; A1: ankle dorsiflexion/plantarflexion at heel strike; A2: ankle dorsiflexion/plantarflexion at midstance phase; A3: ankle dorsiflexion/plantarflexion at toe-off; A4: ankle dorsiflexion/plantarflexion at midswing phase; A5: ankle inversion/eversion at heel strike; A6: ankle inversion/eversion at midstance phase; A7: ankle inversion/eversion at toe-off; A8: ankle inversion/eversion at midswing phase; A9: ankle internal/external rotation at heel strike; A10: ankle internal/external rotation at midstance phase; A11: ankle internal/external rotation at toe-off; A12: ankle internal/external rotation at midswing phase. 
was noted only between Conditions 1 and 3 in the midstance phase. A significant difference was noted between all walking conditions in toe-off $(\mathrm{p}<.05)$.

\section{Discussion}

Spinal movement plays an essential role in maintaining an upright posture and body balance [34] and in reducing the impact transferred to the head during walking [35]. In particular, the waist is an important region for upright human posture; when low back pain is felt, the movements of the pelvis, the spine, and the lower limbs are hindered during walking [4]. Alterations in passive muscle properties may be associated with lower back pain that may be responsible for the altered gait parameters often observed in subjects with back pain [36]. This study helped determine the effects of wearing LSOs with different stiffness levels on the lower-limb kinematics of patients with chronic low back pain during walking, providing basic data for clinical prescriptions and the adaptation of training for the various types of an orthosis. For this purpose, 14 patients with chronic low back pain participated in this study. The average VAS of the participants was 3.07, which was similar to that of the participants in a previous study that examined the gait characteristics of chronic low back pain patients [10,37].

When the kinematic variables of the lower limbs on the sagittal plane were compared, significant differences in the initial contact, midstance, preswing, and midswing in the hip joints and at the midstance and midswing in the knee joints were observed. The flexion of the hip and knee joints decreased more significantly during walking with an LSO than during walking without one. The flexion of the knee joints during walking with an LSO also decreased at the initial contact and preswing, but the difference was not significant. This result may have been caused by a decrease in hip joint flexion when the LSOs limited the pelvic movements. In contrast, Song et al. [31] reported that flexion of the hip and knee joints on the sagittal plane increased markedly to compensate for the limited pelvic movement caused by wearing spinal orthosis. These different results might be explained by differences in the study subjects because, unlike patients with chronic low back pain, healthy people have a mechanism of absorbing ground reaction forces by increasing the flexion of the lower limbs on the sagittal plane when they are limited in their pelvic movements by wearing a spinal orthosis.

The kinematic angles of the lower limbs on the frontal plane showed significant differences at the initial contact, midstance, and preswing of the knee joints. The angles of the genu valgum were reduced in the stance phase more during walking with an LSO than during walking without one. During normal walking, the knee joints are valgus on the frontal plane during the stance phase, and approximately three degrees are added during the loading response [38]. Konz et al. [30] reported that changes produced in the kinematic angles of the lower limbs and the pelvic segments on the frontal plane by wearing spinal orthosis were related directly to decreases in the pelvic ascending/descending obliquity. Thus, the angles of the genu valgum could have been reduced during the stance phase as compensation for the decreased pelvic obliquity from wearing LSOs.

The angles of the lower limbs on the horizontal plane showed significant differences at both the midstance and preswing of the knee joints. The external rotation of the knee joints in the stance phase increased more during walking with an LSO than during walking without one. According to Konz et al. [30], pelvic rotation was reduced markedly by wearing spinal orthosis, and the decreased pelvic angles on the horizontal plane affected the lower-limb kinematics during gait. Thus, the external rotation of the knee joints might have increased in the stance phase as compensation for the decreased pelvic rotation from wearing LSOs.

When the stiffness of the LSOs was compared, 
significant differences were observed between all gait conditions regarding hip flexion/extension at the midswing phase and the knee varus/valgus at toe-off. On the other hand, only wearing a semirigid LSO showed significant differences from walking without an LSO at knee flexion/extension at the midswing phase, knee varus/valgus at heel strike, and knee internal/external rotation at the midstance phase. Cholewicki et al. [32] reported that wearing nonflexible LSOs restricted the trunk $14 \%$ more than wearing a flexible LSO. The present study also used semirigid LSOs, which are significantly stiffer than their flexible counterparts. The limited spinal and pelvic movements affected the lower limbs more during gait.

These results show that patients with chronic low back pain demonstrated significantly different gait characteristics when they wore LSOs compared to when they did not. Flexion of the hip and knee joints on the sagittal plane decreased in subjects who wore LSOs, and the mechanism for moderating vertical ground reaction forces became nonevident. In the lower-limb kinematics on the frontal and horizontal planes, the knee joints compensated for the pelvic obliquity and pelvic rotation that had been limited by the LSOs. The semirigid LSOs showed a larger influence than flexible LSOs did. Previous studies suggested that the short-term effects of wearing a spinal orthosis on gait may be minor, but the long-term effects may be more serious [30]. Overall, walking while wearing an LSO for a long time may decrease the flexibility of the hip and knee joints and may put more load on the knee joint.

This study had some limitations. First, the flexible and semirigid LSOs were off-the-shelf products that came in large, medium, and small sizes considering the body types of the subjects. Differences in the degrees of trunk restriction can occur if LSOs were produced to fit an individual exactly or with different materials. Second, a spinal orthosis is generally designed to be worn for a relatively long time to correct and reposition the vertebral lesions, but the study subjects wore the orthosis only while participating in the experiment. Third, the sample size was small, and the subjects were patients with chronic low back pain. Further studies will be needed to determine if consistent results can be obtained when the subjects undergo spinal surgery. This study did not measure the muscular activities of the lower limbs, and did not evaluate the functional changes caused by wearing LSOs.

\section{Conclusion}

This study examined the effects of wearing LSOs and the effects of different stiffness levels of the orthosis on the gait of patients with chronic low back pain by analyzing the kinematic variables of the lower-limb joints using a formal system for gait examination. The changes in the lower-limb angles on the sagittal, frontal and horizontal planes were measured as the subjects walked with LSOs surrounding the spine and the pelvis. The results showed that flexion of the hip and knee joints on the sagittal plane decreased during walking with LSOs compared to walking without using them. In addition, the genu valgum decreased, and the external rotation of the knee joint increased on both the frontal and the horizontal planes. Stiffer LSOs had greater effects on the kinematics of the lower-limb joints. Hence, it is necessary to prescribe a proper rigid orthosis in clinical prescription and adaptation training for an orthosis. Therefore, to reduce the risk of damage caused by LSO wear in patients with chronic back pain when walking, it is necessary to prescribe a rigid orthosis suitable for the purpose, exercise flexibility in the hip and knee joints, and strengthen the muscles around the knee joint.

\section{References}

[1] Punnett L, Pruss-Utun A, Nelson DI, et al. Estimating the global burden of low back pain attributable to combined occupational exposures. Am J Ind Med. 2005;48(6): 459-69. 
[2] Alexanderson KA, Borg KE, Hensing GK. Sickness absence with low-back, shoulder, or neck diagnoses: an 11-year follow-up regarding gender differences in sickness absence and disability pension. Work (Reading, Mass). 2005;25(2):115-24.

[3] Lahiri S, Markkanen P, Levenstein C. The cost effectiveness of occupational health interventions: preventing occupational back pain. Am J Ind Med. 2005;48(6):515-29.

[4] Cromwell R, Schultz AB, Beck R, et al. Loads on the lumbar trunk during level walking. J Orthop Res. 1989; 7(3):371-7.

[5] Taylor S, Frost H, Taylor A, et al. Reliability and responsiveness of the shuttle walking test in patients with chronic low back pain. Physiother Res Int. 2001;6(3): 170-8.

[6] Vogt L, Pfeifer K, Portscher M, et al. Influences of nonspecific low back pain on three-dimensional lumbar spine kinematics in locomotion. Spine. 2001;26(17): 1910-9.

[7] Al-Obaidi SM, Al-Zoabi B, Al-Shuwaie N, et al. The influence of pain and pain-related fear and disability beliefs on walking velocity in chronic low back pain. Int J Rehabil Res. 2003;26(2):101-8.

[8] Taylor N, Goldie P, Evans O. Movements of the pelvis and lumbar spine during walking in people with acute low back pain. Physiother Res Int. 2004;9(2):74-84.

[9] Vogt L, Pfeifer K, Banzer W. Neuromuscular control of walking with chronic low-back pain. Man Ther. 2003; 8(1):21-8.

[10] Kim K, Ko JY, Lee SY. A study on the characteristics of gait in patients with chronic low back pain. J Korean Soc Phys Ther. 2009;21(2):79-85.

[11] Agabegi SS, Asghar FA, Herkowitz HN. Spinal orthoses. J Am Acad Orthop Surg. 2010;18(11):657-67.

[12] Reyna JR Jr., Leggett SH, Kenney K, et al. The effect of lumbar belts on isolated lumbar muscle. Strength and dynamic capacity. Spine. 1995;20(1):68-73.
[13] Zoia C, Bongetta D, Alicino C, et al. Usefulness of corset adoption after single-level lumbar discectomy: a randomized controlled trial. J Neurosurg Spine. 2018; 28(5):481-5.

[14] Brumagne S, Cordo P, Lysens R, et al. The role of paraspinal muscle spindles in lumbosacral position sense in individuals with and without low back pain. Spine. 2000; 25(8):989-94

[15] Newcomer KL, Laskowski ER, Yu B, et al. Differences in repositioning error among patients with low back pain compared with control subjects. Spine. 2000;25(19): 2488-93

[16] O'Sullivan PB, Burnett A, Floyd AN, et al. Lumbar repositioning deficit in a specific low back pain population. Spine. 2003;28(10):1074-9

[17] Panjabi MM. A hypothesis of chronic back pain: ligament subfailure injuries lead to muscle control dysfunction. Eur Spine J. 2006;15(5):668-76.

[18] Boucher JA, Roy N, Preuss R, et al. The effect of two lumbar belt designs on trunk repositioning sense in people with and without low back pain. Ann Phys Rehabil Med. 2017;60(5):306-11.

[19] Calmels P, Fayolle-Minon I. An update on orthotic devices for the lumbar spine based on a review of the literature. Rev Rhum Engl Ed. 1996;63(4):285-91.

[20] Lariviere C, Caron JM, Preuss R, et al. The effect of different lumbar belt designs on the lumbopelvic rhythm in healthy subjects. BMC Musculoskelet Disord. 2014;15: 307.

[21] van Poppel MN, de Looze MP, Koes BW, et al. Mechanisms of action of lumbar supports: a systematic review. Spine. 2000;25(16):2103-13.

[22] Morrisette DC, Cholewicki J, Logan S, et al. A randomized clinical trial comparing extensible and inextensible lumbosacral orthoses and standard care alone in the management of lower back pain. Spine. 2014;39(21): 1733-42.

[23] Fidler MW, Plasmans CM. The effect of four types of 
support on the segmental mobility of the lumbosacral spine. J Bone Joint Surg Am. 1983;65(7):943-7.

[24] Lantz SA, Schultz AB. Lumbar spine orthosis wearing. I. Restriction of gross body motions. Spine. 1986; 11(8):834-7.

[25] Buchalter D, Kahanovitz N, Viola K, et al. Threedimensional spinal motion measurements. Part 2: A noninvasive assessment of lumbar brace immobilization of the spine. J Spinal Disord. 1988;1(4):284-6.

[26] Grew ND, Deane G. The physical effect of lumbar spinal supports. Prosthet Orthot Int. 1982;6(2):79-87.

[27] Tuong NH, Dansereau J, Maurais G, et al. Threedimensional evaluation of lumbar orthosis effects on spinal behavior. J Rehabil Res Dev. 1998;35(1):34-42.

[28] Kramers-de Quervain IA, Muller R, Stacoff A, et al. Gait analysis in patients with idiopathic scoliosis. Eur Spine J. 2004;13(5):449-56.

[29] Wong MS, Cheng CY, Ng BK, et al. The effect of rigid versus flexible spinal orthosis on the gait pattern of patients with adolescent idiopathic scoliosis. Gait Posture. 2008;27(2):189-95.

[30] Konz R, Fatone S, Gard S. Effect of restricted spinal motion on gait. J Rehabil Res Dev. 2006;43(2):161-70.

[31] Song HN, Kim YM, Kim K. A kinematic analysis of the lower limb with regard to restricted spinal motion during gait. J Phys Ther Sci. 2017;29(1):81-4.

[32] Cholewicki J, Lee AS, Peter Reeves N, et al. Comparison of trunk stiffness provided by different design characteristics of lumbosacral orthoses. Clin Biomech. 2010;25(2):110-4.

[33] Lariviere C, Ludvig D, Kearney R, et al. Identification of intrinsic and reflexive contributions to low-back stiffness: medium-term reliability and construct validity. J Biomech. 2015;48(2):254-61.

[34] Feipel V, De Mesmaeker T, Klein P, et al. Threedimensional kinematics of the lumbar spine during treadmill walking at different speeds. Eur Spine J. 2001; 10(1):16-22.

[35] Smeathers JE. Transient vibrations caused by heel strike. Proc Inst Mech Eng H. 1989;203(4):181-6.

[36] Hines MG, Tillin NA, Luo J, et al. Passive elastic contribution of hip extensors to joint moments during walking in people with low back pain. Clin Biomech. 2018;60:134-40.

[37] Hicks GE, Sions JM, Coyle PC, et al. Altered spatiotemporal characteristics of gait in older adults with chronic low back pain. Gait Posture. 2017;55:172-6.

[38] Perry J, Burnfield JM, Jeong S, et al. Gait analysis: normal and pathological function. Korea. Young Moon. 2012. 